\section{International Scientific Journal Theoretical \& Applied Science}

p-ISSN: 2308-4944 (print) e-ISSN: 2409-0085 (online)

Year: $2014 \quad$ Issue: 12 Volume: 20

Published: $30.12 .2014 \quad \underline{\text { http://www.T-Science.org }}$
Elena Vitalievna Bessarabova Candidate of technical sciences Sebastopol state university, Sebastopol Russia Federation elsev1980@mail.ru

SECTION 21. Pedagogy. Psychology.

Innovations in the field of education.

\title{
PROVE OF EXISTENCE AND CALCULATION OF THE PARAMETERS OF IRRADIATION FIELD
}

Abstract: In the article the principle of occurrence of irradiation field on the eye retina in the process of objects perception is examined. The factors that influence on its occurrence are analysed. Based on the analysis, the main parameters of the field, such as the size of concentration and dissipation, the attenuation degree, the diameter dependence of the eye pupil from the brightness, the influence of diffuse reflectance material on the field size, was calculated.

Key words: irradiation, field, concentration, dissipation, wave front, diffraction, psychophysiology.

Language: Russian

Citation: Bessarabova EV (2014) PROVE OF EXISTENCE AND CALCULATION OF THE PARAMETERS OF IRRADIATION FIELD. ISJ Theoretical \& Applied Science 12 (20): 104-107. doi: http://dx.doi.org/10.15863/TAS.2014.12.20.23

\section{ДОКАЗАТЕЛЬСТВО СУЩЕСТВОВАНИЯ И РАСЧЕТ ПАРАМЕТРОВ ПОЛЯ ИРРАДИАЦИИ}

Аннотация: В статье рассмотрен принции возникновения поля иррадиации на сетчатке глаза при восприятии объектов. Проанализированы факторы, влияющие на его возникновение. На основе проведенного анализа рассчитаны основные параметры поля, такие как: размер конщентрации и рассеивания, степень затухания, зависимость диаметра зрачка от яркости, влияние коэффициента диффузного отражения материала на размер поля.

Ключевые слова: иррадиация, поле, концеентрациия, рассеивание, волновой фронт, дифракция, психофизиология.

Явление иррадиации - это процесс рассеивания изображения на сетчатке глаза, возникающее под влиянием следующих процессов:

- дифрагирования луча отраженного света на круглом отверстии, которым является зрачок человеческого глаза;

- возбуждения и торможения в центральной нервной системе.

Возникновение поля дифракции света на зрачке глаза аналогично процессу рассеивания света на круглом отверстии. Но рассматривая зрачок как один из параметров, влияющих на итоговое поле, следует отметить, что размеры самого зрачка не являются константой, а находятся в зависимости от яркости света. Схема, отражающая совокупность факторов, влияющих на возникновение поля иррадиации, представлена на рисунке 1.
В данной схеме отражены все основные факторы, влияющие на возникновение и распространение поля иррадиации. Рассматривать каждый из этих факторов по отдельности не целесообразно, так как их совокупность - это единая система [3], каждая составляющая которой вносит свой вклад в формирование поля. Но условно всю совокупность факторов можно разделить на две укрупненные группы. Первая группа - это физические характеристики отраженного света и внешних условий, связанных с психофизиологическими параметрами. Вторая группа - исключительно нейрофизиологические характеристики глаза человека [5]. Для того, чтобы установить связь между физической и психофизиологической составляющими [11] рассмотрена одномерная передаточная функция идеальной оптической системы человеческого 
глаза - формула 1. Данная формула преобразует точечное изображение на сетчатке глаза в концентрические кольца $[4,6]$ (рисунок 1) или так называемую функцию рассеяние точки ФРТ. Диаметры таких колец определяются формулами 2 и 3 . На рисунке 1а представлено схематичное изображение поля иррадиации. На рисунке 16 - функция рассеяния точечного изображения на сетчатке глаза. На рисунке 1в представлены обозначения радиусов двух колец рассеивания.

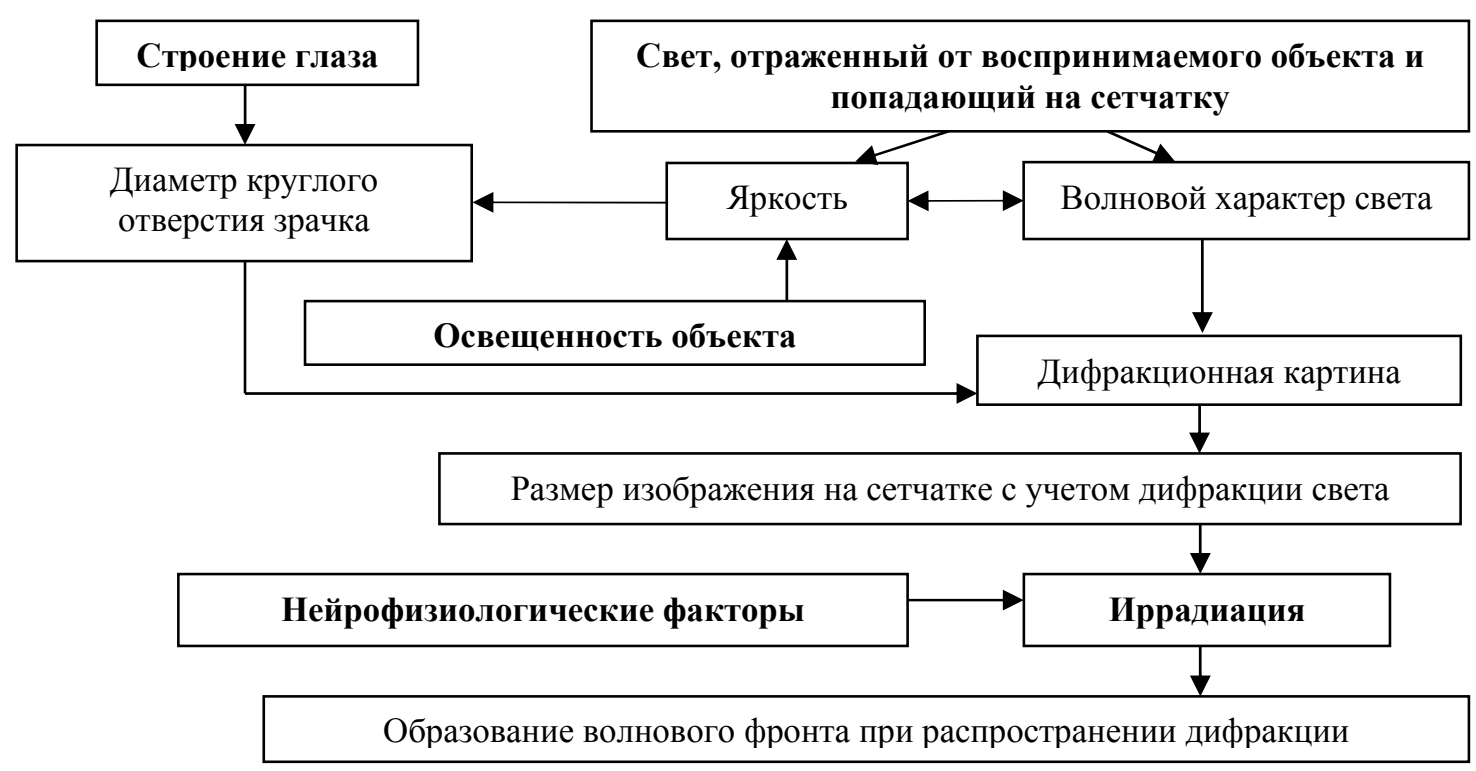

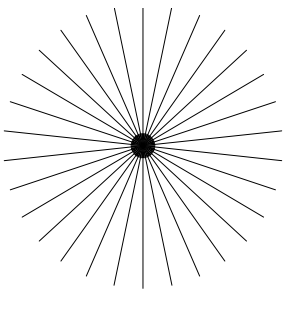

a

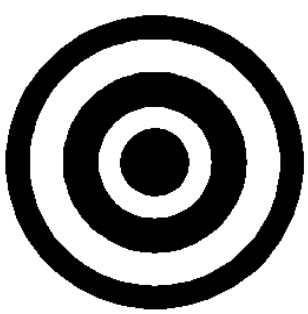

6

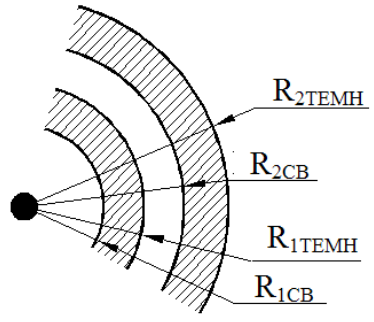

B

Рисунок 1 - Изображение иррадиационного поля.

$$
O(f)=\left(\begin{array}{l}
\frac{2}{\pi}\left(\arccos (u)-u \sqrt{1-u^{2}}\right) \\
0
\end{array}\right)
$$

если $0<\mathrm{u}<1$

$u=\lambda \cdot a^{-1} \cdot d \cdot v$, если $\mathrm{u}>1$

где $\lambda$ - длина волны; $a$ - диаметр зрачка; $d-$ расстояние от хрусталика до сетчатки; $v$ частота.

$D_{1}=2 \beta_{1} \frac{\lambda}{a}=2,44 \frac{\lambda}{a}$;

$$
D_{2}=2 \beta_{2} \frac{\lambda}{a}=4,88 \frac{\lambda}{a}
$$

где $D_{1}, D_{2}$ - диаметры первого и второго колец; коэффициенты, $\beta_{1}, \beta_{2}$-определяющие диаметры рассеивания дифракционной картины с учетом степени затухания.

Картина ФРТ аналогична картине интерференции, которая возникает при дифракции света на круглом отверстии.

Следовательно, картина ФРТ представляет собой чередование светлых и темных колец на сетчатке с темным кольцом в центре. Любое воспринимаемое изображение, каково бы оно не было, линейно или объемно формирует на сетчатке плоские участки возбуждения [2, 9]. Эти участки можно формально разделить на 
линейные и криволинейные. В любом случае волновой фронт будет распространяться вдоль контура изображения в обе стороны от него (радиально). Любой линейный участок возбужденных клеток можно представить совокупностью точек, вовлеченных в процесс формирования изображения, а соответственно совокупностью картин ФРТ.

В зависимости от формы воспринимаемого объекта (в случае, когда контур изображения представляет собой криволинейный участок или ломанную прямую) поле иррадиации [7] может представлять собой следующие локальные варианты распространения:

- линии накладываются одна на другую, сокращая расстояние между собой - случай концентрации - $K$;

- линии увеличивают расстояние между собой - случай рассеивания - $P$.

Примеры локальных характеров распространения иррадиационного поля (концентрация и рассеивание на прямолинейных и криволинейных участках) представлены на рисунке 2.
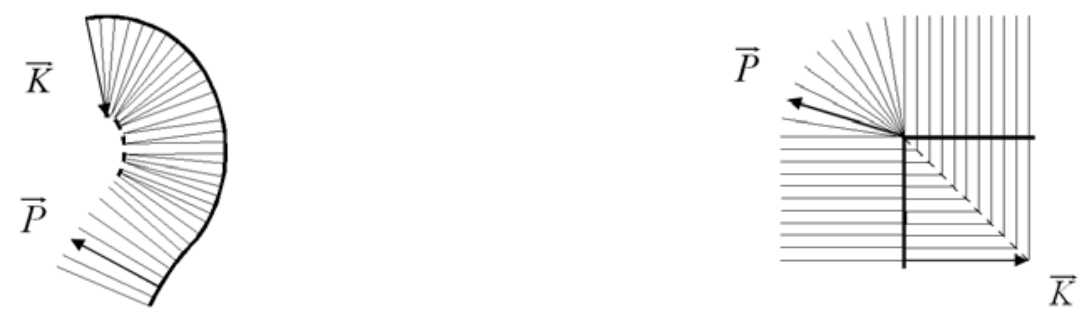

Рисунок 2 - Примеры концентрации и рассеивания линий иррадиационного поля.

При анализе поля иррадиации следует учитывать, что на определенном расстоянии поле затухает, поэтому и локальный характер поля может образовываться только в пределах существования самого поля. Учитывая тот факт, что поле распространяется симметрично от контура объекта в обе стороны формула для расчета концентрации и рассеивания имеет следующий вид:

$$
\vec{K}=\vec{P}=\frac{D_{2}}{2}=R_{2}=2,44 \frac{\lambda}{a}
$$

Диаметр зрачка зависит от яркости воспринимаемого объекта. По экспериментальным данным Вавилова С.И., представленным на рисунке 3, получена формула 5, выражающая логарифмическую зависимость диаметра зрачка от яркости объекта. Основываясь на формуле 5 преобразована формула 4 для расчета размеров иррадиационного поля [1]. В полученной формуле 6 так же учтен коэффициент диффузного отражения материала $\delta$.

$\mathrm{a}, \mathrm{MM}$

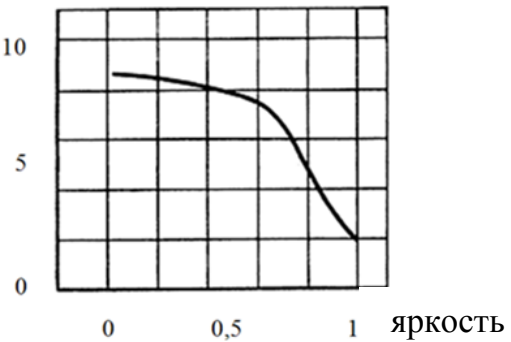

Рисунок 3 - Результата экспериментальных данных зависимости диаметра зрачка от яркости.

$$
\begin{aligned}
& a=1,24\left(\log _{0,9} L\right) \\
& \vec{K}=\vec{P}=\frac{D_{2}}{2}=R_{2}=2 \frac{2,44 \lambda}{1,24 \delta\left(\log _{0,9} L\right)}
\end{aligned}
$$

Данная формула позволяет рассчитать размер поля иррадиации с учетом цвета объекта, его яркости и формирующегося при этом диаметра зрачка, а так же учесть коэффициент диффузного отражения поверхности объекта. Яркость, входящая в формулу, позволяет учесть так же уровень освещенности воспринимаемого объекта, как одну из составляющих формирования яркости [10]. Недостатком 
данной формулы является тот акт, что рассчитать по ней можно размер поля иррадиации, возникающего при восприятии объекта имеющего чисты спектральный цвет.

Формулы 3, 4, 5, 7, отражающие размеры полного поля иррадиации, его рассеивания или концентрации позволяют обосновать зрительные иллюзии, такие как иллюзия размера. Они дают возможность доказать тот факт, что объекты красного цвета кажутся больше, чем фиолетовые при аналогичной яркости. А так же тот факт, что чем ярче объект, тем больше будут казаться его параметры.

Дальнейшее исследование в данной области предполагает изучение взаимного влияния двух соседних полей иррадиации от различных источников. А так же изучение и расчет параметров поля иррадиации, возникающего при восприятии объектов, обладающих реальной цветовой гаммой, отличной от монохромной.

\section{References:}

1. Bessarabova EV (2014) Analiz factorov, vliyayuchich na formirovanie vpechatleniye o vosprinimaemom ob’ecte. Universum. № 8 (10). Available: http://7universum.com/en/philology/archive/it $\mathrm{em} / 1534$

2. Gleizer VD (1985) Zrenie i myschlenie. L., pp. 240 .

3. Korobzeva NA (2005) Teoreticheskie I metodologicheskie osnovi impressivnogo podxoda $\mathrm{k}$ proectirovaniyu odezdi. Moscow, pp. 304.

4. Logvinenko AD (1985) Chyvstvennie osnovi vospriyatiya prostranstva. Moscow, pp. 223.

5. Lyria AP (2006) Osnovi neiropsychologiy. Moscow, pp. 384.
6. Rocc I (1980) Vvedenie v zritelnoe vospriyatie. Moscow, pp.128.

7. Ryyber GE (1985) O zakononernostyiax xydozestvennogo vizyalnogo vospriyatia. Tallin, pp. 344.

8. Sokolov EN (1984) Cvetovyer zrenie. Moscow, pp. 175.

9. Sedler-Smit Y. (2011) Intyicia I mozg. Moscow, pp. 416.

10. Sharonov VV (1961) Svet I cvet. Moscow, pp. 311.

11. Berlyne DE (1971) Aesthetics and Psychobiology. - N.Y.: Appleton-CenturyCrofts, pp. 340. 